\title{
Application of artificial neural network for design: a case of paper helicopter
}

\author{
Tossapol Kiatcharoenpol and Tanaporn Klangpetch \\ Industrial Engineering Department, Faculty of Engineering, King Mongkut's Institute of Technology Ladkrabang, Bangkok, Thailand
}

\begin{abstract}
The design engineering is one of essential work in modern manufacturing environment. The optimization is principal technique to be used widely for searching the solution. However, primary process of optimization is to know the relation between design input parameters and target output. In this work, an artificial neural network (ANN) approach as an intelligent algorithm is proposed to construct the relation and also provides it in form of mathematic modeling. Even though the ANN modeling is so call a backblock due to difficulty to understand complicated equations, it is simply constructed by automate iteration process. A case of paper helicopter is used as an example of the application. The classical 2k Factorial design is used to provide an experiment plan to create training and testing data. 93 experiments are carried out. The architecture of ANN is set according to lowest Mean square error (MSE) of training and testing procedure. The result of 5-10-1 architecture has shown ability to accurately predict output, landing time, with MSE of 0.012. With such a highly quantitative accuracy of results, the developed model using the neural network approach can be used for finding the suitable input parameters to achieve a desired target output. In this case, the design of dimension (A) Depth of cut wing is $1.3 \mathrm{~cm}$., (B) Length of wing is 12.9 $\mathrm{cm}$., (C) Length of body is 9.0, (D) Width of body is $2.0 \mathrm{~cm}$., and (E) Depth of cut body is $0 \mathrm{~cm}$. yield the lowest area of a paper helicopter that can meet the target landing time, $2.85 \pm 5 \%$ second.
\end{abstract}

\section{Introduction}

Application of artificial neural networks (ANN) has been reported by a number of researchers [1-3]. In this study, a Backpropagation neural network has been proposed to map relation between inputs and outputs of a paper helicopter case. The inputs are the dimensions of parts of a helicopter and output are landing times. The optimization based on the ANN model constructed can to be used for designing the suitable dimensions of the helicopter body. To ensure the practical usefulness, a knowledge base for training neural networks was stemmed from experiments carried out over a comprehensive working range of conditions according to full factorial design concept [4]. At the beginning, a brief introduction of neural networks is initially discussed, before the development of neural network models for the intelligent modelling for the estimation of landing time is presented. The suitable features of a paper helicopter that yields the lowest area will be set to achieving the target landing time.

\section{Literature Review}

While the specified literature provides adequate theory on the neural network models studied in this paper, it is useful here to consider the basic theory associated with each of these neural networks with an understanding of the industrial application applied. The architecture and algorithms used by each network to achieve this are significantly different. A brief note on the Backpropagation networks is discussed below.

The standard back propagation network [5-6] comprises 3 layers of processing elements, fully feedforward connected (Fig.1). With the sigmoid on the hidden layer, only the basic equations are :

$$
\begin{aligned}
& y_{k}=\sum_{j=1}^{H} u_{k j} z_{j} \\
& z_{j}=\frac{1}{1+\exp \left(-\theta_{j}\right)} \\
& \theta_{j}=\sum_{i=0}^{N} w_{j i} x_{i}
\end{aligned}
$$

The Least Mean Square error

$$
E=\frac{1}{2} \sum_{k=1}^{M}\left(y_{k}-t_{k}\right)^{2}
$$

All the data are scaled between $0 . .1$ but it can be scaled between $-1 . .1$ to standardise all the inputs with various dimensions. The weights $\mathrm{w}_{\mathrm{ji}}$ and $\mathrm{u}_{\mathrm{kj}}$ are assigned random numbers in the range $-1 . .1$, and a random pair of

\footnotetext{
Corresponding author: tossapol_k@yahoo.com
} 
input / output vectors are picked from the training set. The input vector is fed through the network to get an output vector (feed-forward process); this is then compared with the output vector and an error is found. This error is then passed back through the neural network (back propagation process) to modify the weights using the following equations

$$
\begin{aligned}
& u_{k j}^{n e w}=u_{k j}^{\text {old }}+\Delta u_{k j} \\
& w_{j i}^{n e w}=w_{j i}^{\text {old }}+\Delta w_{j i}
\end{aligned}
$$

The gradient descent optimization technique is used to calculate the change in each weight. This is then repeated by picking another random pair of input / output vectors and continuing until the error is at a minimum. This was done 1.5 million times which was sufficient for the network to reach a suitable minimum.

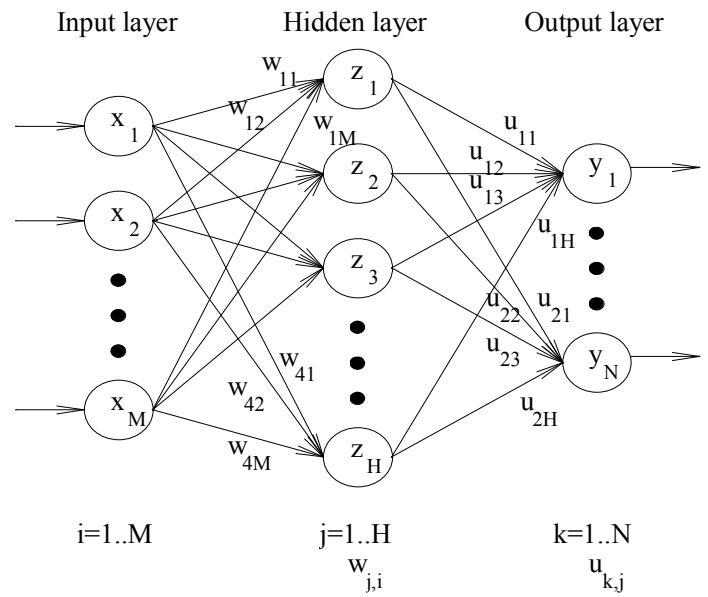

Fig. 1. Multi-layered Back Propagation Neural Network

Momentum can be used to decrease times in training and the chance of the network getting stuck in a shallow minimum. This is done by accelerating the convergence of the error but is not applied in this situation.

\section{Develop of Database}

The ANN is applied to build up a model of a paper helicopter case. There are five inputs: (A) Depth of cut wing, (B) Length of wing, (C) Length of body, (D) Width of body, and (E) Depth of cut body in Figure 2. The output is landing time in second where a paper helicopter is released from eight feet height.

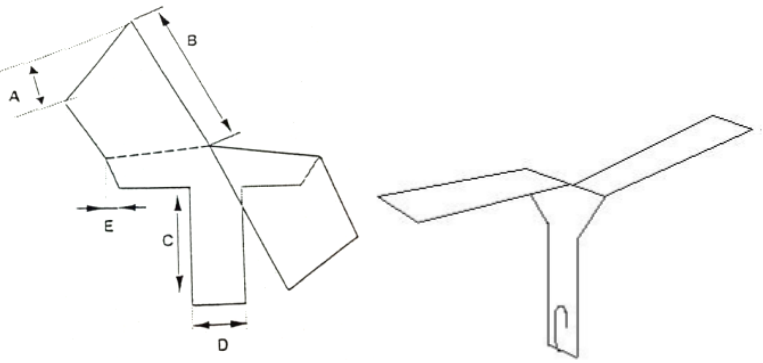

Fig. 2. Factors of A Paper Helicopter

Full Factorial 2k is used to design experiment plan to construct the database. Five inputs or factors are conditioned at two levels of values as shown in Table 1.

\begin{tabular}{|c|c|c|c|}
\hline \multirow{2}{*}{$\begin{array}{l}\text { Input or } \\
\text { Factor }\end{array}$} & \multicolumn{2}{|c|}{ Level } & \multirow[b]{2}{*}{ unit } \\
\hline & Low (-) & $\begin{array}{l}\text { High } \\
(+)\end{array}$ & \\
\hline $\begin{array}{l}\text { (A) Depth of } \\
\text { cut wing }\end{array}$ & 1.0 & 2.0 & $\mathrm{~cm}$. \\
\hline $\begin{array}{l}\text { (B) Length of } \\
\text { wing }\end{array}$ & 11.0 & 13.0 & $\mathrm{~cm}$. \\
\hline $\begin{array}{l}\text { (C) Length of } \\
\text { body }\end{array}$ & 8.0 & 9.0 & $\mathrm{~cm}$. \\
\hline $\begin{array}{l}\text { (D) Width of } \\
\text { body }\end{array}$ & 2.0 & 3.0 & $\mathrm{~cm}$. \\
\hline $\begin{array}{l}\text { (E) Depth of } \\
\text { cut body }\end{array}$ & 0.0 & 1.0 & $\mathrm{~cm}$. \\
\hline
\end{tabular}

Table 1. Factors and Levels

The experiments of 32 various conditions with three replications were carried out. The overall 96 data are obtained to be the database for training and testing ANN.

\section{Training and Testing ANN}

Back propagation ANN with one hidden layer is used for modeling. The number of neurons in input layer is set to five neurons according to the number of inputs and for the output Layer, the number of neurons are set to one. The number of neurons in hidden layer is basically determined, which based on the minimum error of testing data. The mean square error (MSE) is used in trail of neurons from 7 to 13 neurons. The results are shown in Table 2.

The Backpropagation ANN of 5-10-1 yielding the minimum of error is used in this case of paper helicopter. The relation of output or landing time and inputs of dimensions are modeling even though the mathematical model is hardly depicted. The prediction of landing time could be estimated based on the ANN black box. 
Table 2. Factors and Levels

\begin{tabular}{|l|r|c|c|c|}
\hline \multirow{2}{*}{ No. } & \multicolumn{3}{|c|}{ Number of Neurons } & \multirow{2}{*}{ MSE } \\
\cline { 2 - 4 } & Input & Hidden & Output & \\
\hline 1 & 5 & 7 & 1 & 0.031065 \\
\hline 2 & 5 & 8 & 1 & 0.029954 \\
\hline 3 & 5 & 9 & 1 & 0.023788 \\
\hline $\mathbf{4}$ & $\mathbf{5}$ & $\mathbf{1 0}$ & $\mathbf{1}$ & $\mathbf{0 . 0 1 2 2 8 3}$ \\
\hline 5 & 5 & 11 & 1 & 0.019383 \\
\hline 6 & 5 & 12 & 1 & 0.029194 \\
\hline 7 & 5 & 13 & 1 & 0.021212 \\
\hline
\end{tabular}

\section{Design of Suitable Condition}

\subsection{Selection of Suitable Condition}

The target of Landing Time is set at $2.90 \pm 0.05$. Then the conditions of the five inputs have to be searched in order to meet the target. Since five inputs are the dimension of a paper helicopter, the condition of five inputs providing the lowest area is suitable condition. The 307,461 combination of five inputs are put in to ANN Model constructed as shown in Fig. 3. The results are presented in Table 3.

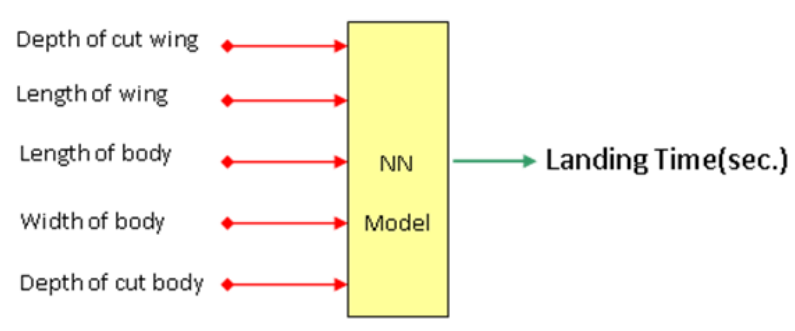

Fig. 3. ANN model for Estimating Landing Time

Table 3. Results of Searching Suitable Conditions

\begin{tabular}{|l|l|l|l|l|l|l|l|}
\hline $\begin{array}{l}\text { No } \\
\text { A }\end{array}$ & $\mathrm{B}$ & $\mathrm{C}$ & $\mathrm{D}$ & $\mathrm{E}$ & Time & Area \\
\hline 1 & 1.1 & 12.9 & 9.0 & 2.0 & 0.0 & 2.852 & 110.1 \\
\hline 2 & 1.2 & 12.9 & 9.0 & 2.0 & 0.0 & 2.852 & 109.8 \\
\hline $\mathbf{3}$ & $\mathbf{1 . 3}$ & $\mathbf{1 2 . 9}$ & $\mathbf{9 . 0}$ & $\mathbf{2 . 0}$ & $\mathbf{0 . 0}$ & $\mathbf{2 . 8 5 0}$ & $\mathbf{1 0 9 . 5}$ \\
\hline 4 & 1.0 & 13.0 & 9.0 & 2.0 & 0.0 & 2.854 & 111.0 \\
\hline 5 & 1.1 & 13.0 & 9.0 & 2.0 & 0.0 & 2.856 & 110.7 \\
\hline 6 & 1.2 & 13.0 & 9.0 & 2.0 & 0.0 & 2.855 & 110.4 \\
\hline 7 & 1.3 & 13.0 & 9.0 & 2.0 & 0.0 & 2.853 & 110.1 \\
\hline 8 & 1.0 & 13.0 & 9.0 & 2.1 & 0.0 & 2.851 & 111.9 \\
\hline
\end{tabular}

The suitable condition should be the number 3; (A) Depth of cut wing is $1.3 \mathrm{~cm}$., (B) Length of wing is 12.9 cm., (C) Length of body is 9.0, (D) Width of body is 2.0 $\mathrm{cm}$., and (E) Depth of cut body is $0 \mathrm{~cm}$. The lowest area of a paper helicopter is $109.5 \mathrm{~cm} 2$.

\subsection{Verification of Result}

The suitable condition of design inputs are tested in order to confirm that could yield the output, landing time 2.85 second within $\pm 5 \%$. The twenty experiments were carried out and the experimental results are shown in Fig. 4.

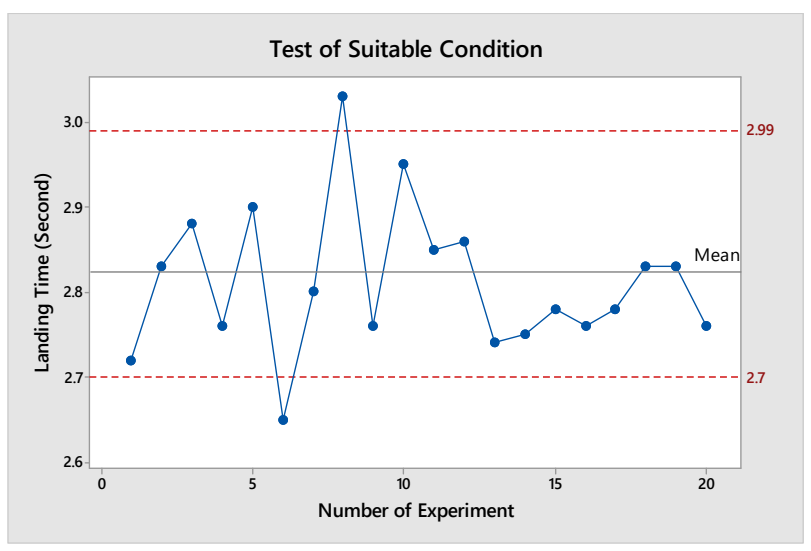

Fig. 4. Experimental Results of Suitable Condition

In suitable condition, $\mathrm{A}=1.3, \mathrm{~B}=12.9, \mathrm{C}=9.0$, $\mathrm{D}=2.0$ and $\mathrm{E}=0$, almost of twenty data are between 2.85 $+5 \%(2.70-2.99)$ second. The arithmatic mean is 2.81 second when compare to 2.85 second from the prediction of the ANN. The error is about $1.4 \%$, which support the accuracy of developed ANN model.

\section{Conclusion}

A Backpropagation ANN is studied to apply in modeling a case of paper helicopter. The landing time as an output is targeted in order to find suitable dimensions of a paper helicopter. Five dimensions are chosen as inputs in this design. 2k Full factorial design is used to provide the experimental plan to build a database for training and testing ANN. The 32 experimental conditions with three replicates are carried out to create 96 databases. The architecture of ANN is determined based on providing the lowest error of test data set. After training and testing procedure, the ANN (5-10-1) with one hidden layer is set and used to model relation between the output and inputs. The design of dimension (A) Depth of cut wing is $1.3 \mathrm{~cm}$., (B) Length of wing is $12.9 \mathrm{~cm}$., (C) Length of body is 9.0 , (D) Width of body is $2.0 \mathrm{~cm}$., and (E) Depth of cut body is $0 \mathrm{~cm}$. yield the lowest area of a paper helicopter that can meet the target landing time, $2.85 \pm$ $5 \%$ second. This ANN for modeling in this case would be useful to mapping input and output for selecting suitable condition. 


\section{References}

1. V. Karri, and T. Kiatcharoenpol, A monitoring system of drill wear states using a hybrid neural network, Materials Science Forum (2004)

2. V. Karri, and T. Kiatcharoenpol, Tool condition monitoring in drilling using artificial neural networks, Lecture Notes in Computer Science (including subseries Lecture Notes in Artificial Intelligence and Lecture Notes in Bioinformatics) (2003)

3. V. Karri, and T. Kiatcharoenpol, Prediction of Internal Surface Roughness in Drilling Using Three Feedforward Neural Networks - A Comparison, 9th International Conference On Neural Information Processing, Singapore, (2002)
4. C. Montgomery Douglas, Design and analysis of experiments. 8.th ed. Hoboken, NJ : Wiley, (2013)

5. F. Ortega-Zamorano, J. M. Jerez, D. Urda Muñoz, R. M. Luque-Baena, L. Franco, Efficient Implementation of the Backpropagation Algorithm in FPGAs and Microcontrollers IEEE Transactions on Neural Networks and Learning Systems (2016)

6. N. V. Irukulapati, D. Marsella, P. Johannisson, E. Agrell, M. Secondini, H. Wymeersch, Stochastic Digital Backpropagation With Residual Memory Compensation, Journal of Lightwave Technology (2016) 